\title{
year posterior corneal changes after Bowman Layer Transplant for keratoconus
}

European Journal of Ophthalmology $1-5$

(C) The Author(s) 2021 Article reuse guidelines:

sagepub.com/journals-permissions DOI: 10.1 | $77 / 1$ | 20672/21 1054730 journals.sagepub.com/home/ejo

(S)AGE

\author{
Abdo Karim Tourkmani' (D), Conor Lyons', \\ Parwez N. Hossain ${ }^{2}$, Aristides Konstantopoulos', \\ David F. Anderson ${ }^{2}$ and Jorge L. Alio ${ }^{3}$
}

\begin{abstract}
Introduction: To report the posterior corneal changes after Bowman Layer Transplant for keratoconus in a tertiary hospital in the UK.

Methods: 5 eyes of 5 patients receiving Bowman Layer Transplant for advanced keratoconus in Royal Gwent Hospital (Newport, UK) were included. Pre and postoperative posterior corneal astigmatism, posterior Kmean, and back surface elevation were analysed.

Results: No significant changes were seen in the posterior corneal astigmatism, posterior Kmean, or back surface elevation between the pre- and postoperative period.

Conclusion: This results would support the idea that the corneal changes seen after Bowman Layer Transplant are mainly in the anterior corneal surface.
\end{abstract}

\section{Keywords}

Keratoconus, bowman Layer Transplant, posterior Cornea

Date received: 20 July 2021 ; accepted 24 September 202 I

\section{Introduction}

Kearotoconus is an ectatic corneal disorder which can lead to corneal thinning and protrusion, hence impairing visual function. ${ }^{1}$ Treatment for this condition varies from spectacle or contact lens correction, to surgery such as Intracorneal Ring Segments (ICRS), ${ }^{2}$ Corneal Cross Linking (CXL), ${ }^{3}$ Corneal Allogeneic Ring Segments (CAIRS), ${ }^{4}$ Femtosecond Intra Lenticular Implantation (FILI), ${ }^{5}$ Intracorneal Regeneratice Procedures (ICRP), ${ }^{6}$ Bowman Layer Transplant (BLT), ${ }^{7}$ Deep Anterior Lamellar Keratoplasty (DALK), and Penetrating Keratoplasty (PKP). ${ }^{8}$

We recently published our results with BLT and the corneal changes in the central and paracentral region. ${ }^{9}$ We demonstrated greater keratometric reduction with this surgery in the paracentral area compared to the central area, by measuring the Equivalent Keratometry Readings (EKR) from Holladay report, which intergrates both anterior and posterior corneal surfaces.

This would raise the question as to whether the posterior corneal changes are totally or partially responsible of such a difference; or whether they are not relevant at all.
Hence, the posterior corneal changes on their own deserve a more detailed investigation.

We believe that it is of relevance to understand the anatomical changes that arise in the posterior corneal surface, if any, following BLT. Hence, we herein report the posterior corneal changes after BLT in a specialised eye unit in South Wales.

\section{Methods}

This retrospective case series review was performed in Royal Gwent Hospital, Newport, Wales, UK. Keratoconic patients with stage III - IV keratoconus using Amsler

\footnotetext{
'Royal Gwent Hospital, Eye Unit. Newport, Wales, UK

${ }^{2}$ Southampton General Hospital, Eye Unit. Southampton, England, UK

${ }^{3}$ Vissum Instituto Oftalmologico, Alicante, Spain

Corresponding author:

Abdo Karim Tourkmani, Cornea, Cataract and Anterior Segment, Royal Gwent Hospital, Cardiff Rd, Newport NP20 2UB, UK.

Email: Karim.Tourkmani2@wales.nhs.uk
} 
Krumeich classification and unable to tolerate contact lens correction of any kind (rigid, piggy back or hybrid) were identified in the corneal clinic. Examination included slit lamp evaluation, tonometry, dilated funduscopy, and corneal topography / tomography (Pentacam).

Patients fulfilling the above criteria were offered PKP, DALK or BLT and were informed about differences, risks and benefits of each procedure.

8 eyes of 8 patients were scheduled for BLT between November 2018 and January 2020, with 5 cases having successful, uncomplicated surgery. All surgeries were performed by an experienced corneal surgeon, however in his early learning curve of BLT procedure (AKT). Subtle modifications to the original Bowman Layer (BL) graft harvesting technique were introduced as those helped the surgeon to obtain a BL graft more successfully in the wetlab training. Those graft-harvest protocol changes have been reported in an earlier work of this group. ${ }^{9}$ Basically, after mounting the donor corneoscleral rim in an artificial anterior chamber and removing the epithelium, gentle scoring of the periphery of $\mathrm{BL}$ was performed with a $30 \mathrm{~g}$ needle. Then the blunt end of either a McPhersons forceps or a Morlett spatula were used to gently "scrape" the edge of the scoring mark, which helped to lift a BL "flap". This was performed 360 degrees and allowed for an easier grasp of BL, therefore an easier peeling off of BL. Thee peeling process was performed with a Moorfields forceps which would provide a greater area of contact than a McPherson forceps, hence distributing the tension over an area rather than focalizing all the tension on a point, possibly reducing the risk of $\mathrm{BL}$ tears (Figures 1 and 2).

All of the cases completed a 1-year follow up postoperative, however postoperative follow up visits in between did not follow a standardised regime, since hospital attention had to be modified due to COVID pandemic. All cases had 1 day, 1 week and 1-to-3 months review, then another review between 10 and 14 months after surgery (which was deemed the 1-year follow up visit). VA, slit lamp examination, and corneal topography / tomography were performed at each visit. Rigid contact lens trial was performed after the 3 month postoperative period. Visual outcomes and contact lens tolerance postoperative were reported in our earlier paper. ${ }^{9}$ For the purpose of this paper we will concentrate on the posterior corneal changes postoperative.

Of the 5 successful cases included in this study, we analysed the maximum point of back surface elevation, back surface Kmean, and posterior corneal astigmatism. All these anatomical values were obtained from Pentacam. Endothelial cell density was not analysed given the lack of specular microscopy in our department. Excel (Microsoft) was used for statistics (mean and standard deviation).

\section{Results}

Of the 8 eyes of 8 patients receiving BLT, 3 surgeries were complicated by either microperforation of host cornea $(1$

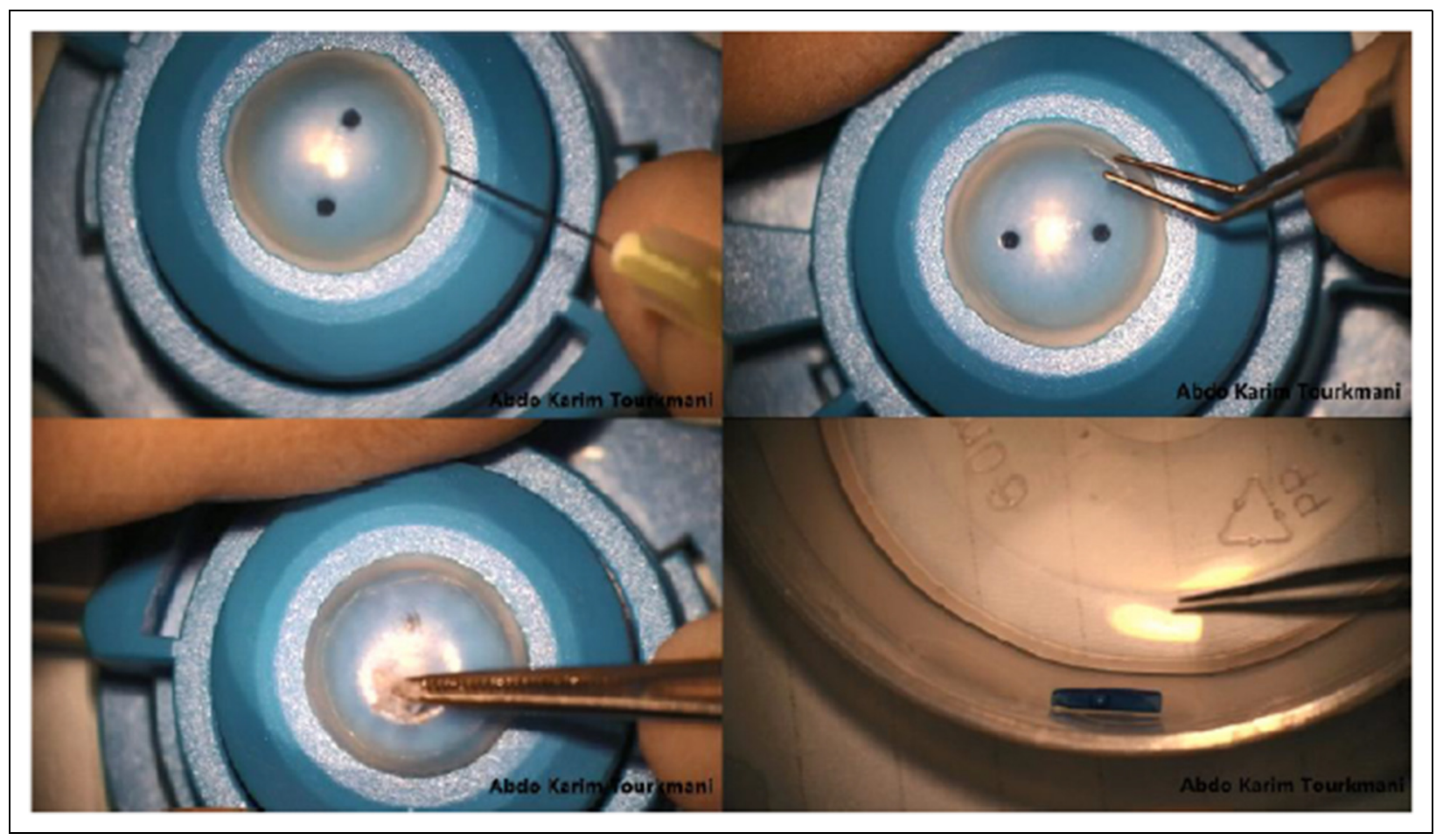

Figure I. BL graft harvest. Top left, BL scoring with 30g needle. Top right, BL “flap” lift 360 degrees with McPherson's forceps. Bottom left, peeling of $\mathrm{BL}$ graft with Moorfields forceps. Bottom right, $8 \mathrm{~mm}$ BL roll stained with trypan blue. 


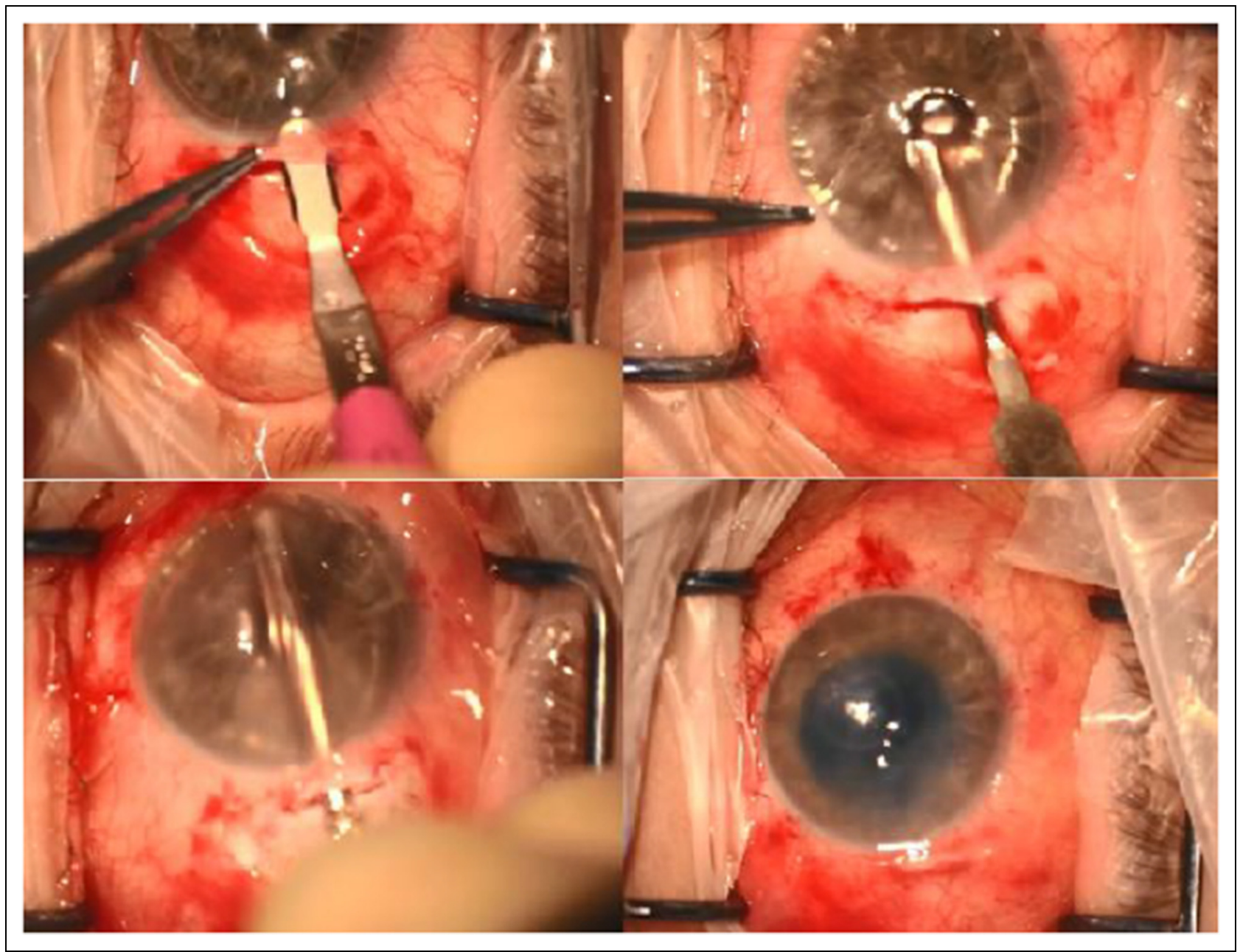

Figure 2. BLT surgery. Top left, peritomy and corneoscleral tunnel. Top right, midstromal pocket dissection. Note reflection under spatula from air - endothelium interface, showing depth of dissection. Bottom left, limbus to limbus midstromal pocket dissection. Bottom right, BL graft introduced in host corneal pocket and unfolded.

Table I. Individual and mean value for maximum back elevation (microns) pre and postoperative.

\begin{tabular}{lll}
\hline $\begin{array}{l}\text { Maximum back } \\
\text { elevation (microns) }\end{array}$ & Pre operative & Postoperative \\
\hline Case I & 152 & 97 \\
Case 2 & 86 & 95 \\
Case 3 & 82 & 71 \\
Case 4 & 131 & 109 \\
Case 5 & 136 & 140 \\
Mean (SD) & $117.4(31.49)$ & $102.4(25.13)$ \\
\hline
\end{tabular}

case, $12.5 \%$ ), or donor tissue wastage (1 case due to a tear in donor tissue, and one case due to donor tissue being thicker than intended, 25\%), within standards compared to previous reports. ${ }^{7}$

The remaining 5 successful cases have been included in this study. 3 were left and 2 were right eyes.
The mean value of maximum back elevation in the back elevation map preoperatively was 117.4 microns (SD 31.49) and 102.4 microns (SD 25.13) postoperatively. Mean posterior corneal astigmatism (PCA) was $1.1 \mathrm{D}$ preoperatively (SD $0.34)$ and $1.26 \mathrm{D}$ (SD 0.58) postoperatively. The mean value of posterior Kmean (PKm) was -10.04 D (SD 1.6) preoperatively and $-10.06 \mathrm{D}$ (SD 1.38) postoperatively.

Tables 1, 2 and 3 show the values for the abovementioned parameters for each case individually, alongside with posterior $\mathrm{K} 1$ and $\mathrm{K} 2$ values.

\section{Discussion}

Keratoconus can be a significantly debilitating corneal disorder, with visual impairment requiring corneal surgery for rehabilitation. $^{1,10}$

Most of studies reporting the topographic / tomographic and / or refractive results of the various techniques that can 
Table 2. Posterior corneal astigmatism (PCA) and posterior keratometry readings (PKI, PK2) pre and postoperative.

\begin{tabular}{lllllll}
\hline PCA and PKs (D) & PKI pre & PK2 pre & PKI post & PK2 post & PCA pre & PCA post \\
\hline Case I & -9.6 & $-10.8 @ 8.5$ & -9.9 & -10.9 @ I78 & 1.2 & 1 \\
Case 2 & -8.2 & $-8.7 @ 81$ & -8.5 & $-9.1 @ 80$ & 0.5 & 0.6 \\
Case 3 & -7.8 & $-9.2 @ 85$ & -7.9 & $-9.2 @ 84$ & 1.4 & 1.3 \\
Case 4 & -10.9 & $-12.1 @ 53$ & -10.2 & $-11.4 @ 36$ & 1.2 & 1.2 \\
Case 5 & -11.1 & $-12.3 @ 49$ & -10.8 & $-13 @ 86$ & 1.2 & 2.2 \\
Mean (SD) & & & & & $1.1(0.34)$ & $1.26(0.58)$ \\
\hline
\end{tabular}

Table 3. Posterior kmean pre and postoperative.

\begin{tabular}{lcc}
\hline Posterior Km (D) & Preoperative & Postoperative \\
\hline Case I & -10.2 & -10.4 \\
Case 2 & -8.4 & -8.8 \\
Case 3 & -8.4 & -8.5 \\
Case 4 & -11.5 & -10.8 \\
Case 5 & -11.7 & -11.8 \\
Mean (SD) & $-10.4(1.6)$ & $-10.6(1.38)$ \\
\hline
\end{tabular}

be used to tackle this condition concentrate on the front corneal topography, which usually covers the central 3 $\mathrm{mm}$ of the cornea. ${ }^{2-8}$

We recently reported the corneal changes after BLT for keratoconus in the central and paracentral cornea, since an $8 \mathrm{~mm}$ (or beyond) Bowman Layer graft would cover at least the central $6 \mathrm{~mm}$ of the cornea, even for steeper corneas. $^{9}$

We are also more aware of the importance of the posterior corneal surface and posterior corneal astigmatism for corneal and other refractive procedures, such as modern cataract surgery. ${ }^{11}$ Without BLT being a refractive procedure - in the traditional sense of aiming for a target of plano refraction after the procedure-, the main goals of the procedure are to halt / delay keratoconic progression and modify corneal shape so that patients are able to regain contact lens tolerance. In modifying corneal shape, there are by definition refractive corneal changes, which would contribute to some visual improvement following surgery; ${ }^{7,9}$ whether with -for most patients- or without correction -for some-. Whereas previous studies regarding the topographical / tomographical outcomes after BLT concentrate on the front topography, ${ }^{7}$ to our knowledge there are no studies other than our previous one that integrate ${ }^{9}$ (in a unified manner) the posterior corneal changes following BLT; and only one that specifically analyses the posterior corneal changes following BLT. ${ }^{12}$ For our previous work, we hence decided to study the topographical / tomographical changes in the Holladay report of Pentacam, since these would 1) analyse different sectors of the cornea covering an area broader than the central $3 \mathrm{~mm}$ and 2) integrate in its EKRs both the anterior and posterior corneal surfaces.
We found that the corneal changes were more significant in the paracentral than the central area. In using the EKRs, this raised now the question as to whether these changes were related to the posterior corneal surface, or whether those would still be related to the anterior surface alone.

As such, a more detailed investigation of the posterior surface on its own was warranted.

Our results in this study show a mild topographical reduction in the back surface elevation, however there was no identifiable linear relation between the amount of corneal flattening reported in our previous work ${ }^{9}$ and the amount of decrease in back surface elevation.

With regards to PCA and PKm, there was a slight increase in mean PCA postoperatively, whereas PKm remained largely unchanged after the procedure.

In our study we found no significant changes in the posterior corneal parameters that we analysed, in this cohort of patients with progressive $\mathrm{KC}$ whom underwent BLT. The findings of this paper would suggest that the anatomical improvement following BLT happens mainly in the front surface of the cornea and that the contribution of the posterior corneal changes is probably negligible.

This, alongside with our previous work, would suggest that the anatomical corneal changes after BLT happen mainly in the central and paracentral front surface of the cornea, with the posterior surface of the cornea remaining largely similar to preoperatively.

Potential limitations of this study are the small number of patients involved, and lack of standardization in the post operative follow up regime (due to COVID pandemic). As for donor corneal tissue wastage, this event can be avoided by ordering pre-stripped Bowman Layer tissue.

Further studies with a larger number of patients are needed to investigate this topic.

\section{Ethics approval}

Formal ethics approval from the University Research Ethics Board was not required for the completion of this case report.

\section{Consent to participate}

Signed consent was obtained from the patient for the use of their medical records in writing this case report. A copy of the written 
consent is available for review by the Editor-in-Chief of this journal under request

\section{Authors' contributions}

A. K. Tourkmani was the primary consultant caring for the patient. A. K.Tourkmani was the consultant surgeon who completed all surgeries reported and completed the draft manuscript. All authors contributed to data collection, reviewed and approved the final manuscript.

\section{Availability of data and materials}

The datasets generated and/or analysed during the current study are available from the corresponding author on reasonable request

\section{Declaration of Conflicting Interests}

The author(s) declared no potential conflicts of interest with respect to the research, authorship, and/or publication of this article.

\section{Funding}

The author(s) received no financial support for the research, authorship and/or publication of this article.

\section{ORCID iDs}

Abdo Karim Tourkmani (iD https://orcid.org/0000-0001-7356$573 \mathrm{X}$

Jorge L. Alio (ID) https://orcid.org/0000-0002-8082-1751

\section{Supplemental material}

Supplemental material for this article is available online.

\section{References}

1. Mas Tur V, MacGregor C, Jayaswal R, et al. A review of keratoconus: diagnosis, pathophysiology, and genetics. Surv Ophthalmol. 2017 Nov-Dec; 62: 770-783. Epub 2017 Jul 6. PMID: 28688894.

2. Siganos D, Ferrara $P$, Chatzinikolas K, et al. Ferrara intrastromal corneal rings for the correction of keratoconus. J Cataract Refract Surg 2002 Nov; 28: 1947-1951. PMID: 12457667.
3. Kobashi $\mathrm{H}$ and Rong SS. Corneal collagen cross-linking for keratoconus: systematic review. Biomed Res Int 2017; 2017: 8145651. Epub 2017 Jun 11. PMID: 28691035; PMCID: PMC5485290.

4. Jacob S, Patel SR, Agarwal A, et al. Corneal allogenic intrastromal ring segments (CAIRS) combined With corneal crosslinking for keratoconus. J Refract Surg 2018 May 1; 34: 296303. PMID: 29738584.

5. Ganesh S and Brar S. Femtosecond intrastromal lenticular implantation combined With accelerated collagen crosslinking for the treatment of keratoconus-initial clinical result in 6 eyes. Cornea 2015 Oct; 34: 1331-1339. PMID: 26252741

6. Alió Del Barrio JL, Arnalich-Montiel F, De Miguel MP, et al. Corneal stroma regeneration: preclinical studies. Exp Eye Res. 2020 Oct; 24: 108314. Epub ahead of print. PMID: 33164825 .

7. Dragnea DC, Birbal RS, Ham L, et al. Bowman layer transplantation in the treatment of keratoconus. Eye Vis (Lond 2018 Sep 12; 5: 3-14. doi: 10.1016/j.clae.2016.10.001. Epub 2016 Oct 29. PMID: 27802912.

8. Henein $\mathrm{C}$ and Nanavaty MA. Systematic review comparing penetrating keratoplasty and deep anterior lamellar keratoplasty for management of keratoconus. Cont Lens Anterior Eye. 2017; 40(1): 3-14. doi: 10.1016/j.clae.2016.10.001. Epub 2016 Oct 174 29. PMID: 27802912.

9. Tourkmani AK, Mohammad T, mcCance E, et al. 1 Year front versus central and paracentral corneal changes after bowman layer transplant for keratoconus. Cornea 2021 Apr 14. doi: 10.1097/ICO.0000000000002733. Epub ahead of print. PMID: 33859089 .

10. Tu KL, Tourkmani AK and Srinivas S. Interrelationships between 3 keratoconic cone parameters. Cornea 2017 Sep; 36: 1051-1053. PMID: 28704318.

11. LaHood BR and Goggin M. Measurement of posterior corneal astigmatism by the IOLMaster 700. J Refract Surg 2018 May 1; 34: 331-336. PMID: 29738589.

12. van Dijk K, Liarakos VS, Parker J, et al. Bowman layer transplantation to reduce and stabilize progressive, advanced keratoconus. Ophthalmology 2015 May; 122: 909-917. Epub 2015 Jan 14. PMID: 25596620. 